\title{
Sciatica in the Young
}

\author{
Sim Sai Tin ${ }^{1}$, Viroj Wiwanitkit ${ }^{2}$ \\ ${ }^{1}$ Medical Center, Shantou, China \\ ${ }^{2}$ Hainan Medical University, Haikou,China
}

Dear Editor,

\section{References}

We would like to discuss on the publication entitled "sciatica in the young [1]." Omidi-Kashani et al. [1] reported a case of "sciatica in a five-year-old boy" and noted that "lumbar osteoid osteoma should be suspected as the cause of low back pain or sciatalgia in any young patient." Sciatica in the young, especially in children, is rare. Lumbar osteoid osteoma is an important condition which can be systematic. Investigation on other parts of the child to find any abnormal osteoma is required [2]. Nevertheless, there are also other uncommon conditions that should be considered. Sciatica in children is generally due to lumbar disc herniation as a result of accidental injury [3]. Sometimes, the uncommon conditions could be the cause of sciatica in the children. Good examples include Garre's chronic sclerosing osteomyelitis [4] and pyomyositis [5]. Sciatica as a complication of missed injection procedure has also been reported [6]. To differentiate these conditions, complete history, clinical examination, imaging investigation, and laboratory tests should be performed.

\section{Conflict of Interest}

No potential conflict of interest relevant to this article was reported.
1. Omidi-Kashani F, Hasankhani EG, Rafeemanesh E. Sciatica in a five-year-old boy. Asian Spine J 2014;8: 357-60.

2. Athwal P, Stock H. Osteoid osteoma: a pictorial review. Conn Med 2014;78:233-5.

3. Martinez-Lage JF, Fernandez Cornejo V, Lopez F, Poza M. Lumbar disc herniation in early childhood: case report and literature review. Childs Nerv Syst 2003;19:258-60.

4. Franco-Jimenez S, Romero-Aguilar JF, Bervel-Clemente $\mathrm{S}$, et al. Garre's chronic sclerosing osteomyelitis with sacral involvement in a child. Rev Esp Cir Ortop Traumatol 2013;57:145-9.

5. Toda T, Koda M, Rokkaku T, et al. Sciatica caused by pyomyositis of the piriformis muscle in a pediatric patient. Orthopedics 2013;36:e257-9.

6. Topuz K, Kutlay M, Simsek H, Atabey C, Demircan $\mathrm{M}$, Senol Guney M. Early surgical treatment protocol for sciatic nerve injury due to injection--a retrospective study. Br J Neurosurg 2011;25:509-15.

Received Sep 5, 2014; Accepted Sep 6, 2014

Corresponding author: Sim Sai Tin

Medical Center, Shantou, China

Tel: +86-754-2564136, Fax: +86-754-2564136, E-mail: simsaitin@gmail.com 